\title{
Uterine Rupture and Pregnancy Current Vision: Scientific Evidence and Consensus with the Patient
}

\section{Inmmaculada Nicolás Perea*}

Department of Gynaecology and Obstetrics, Hospital El Pilar, Barcelona, Spain

*Corresponding author: Inmmaculada Nicolás Perea, Department of Gynaecology and Obstetrics, Hospital El Pilar, Barcelona, Spain, E-mail: innipe@hotmail.com

Received date: July 14, 2016; Accepted date: August 03, 2016; Published date: August 10, 2016

Citation: Inmmaculada Nicolás Perea (2016) Uterine Rupture and Pregnancy Current Vision: Scientific Evidence and Consensus with the Patient. Reproductive Immunol Open Acc 1:19. doi: 10.21767/2476-1974.100019

Copyright: (c) 2016 Nicolás Perea I. This is an open-access article distributed under the terms of the Creative Commons Attribution License, which permits unrestricted use, distribution, and reproduction in any medium, provided the original author and source are credited.

\begin{abstract}
:
Uterine rupture during pregnancy is the disruption of the uterine wall. It is a rare complication but it is associated with high maternal and perinatal morbidity and mortality. This has a variable incidence between $0.02-0.08 \%$ of pregnancies. There are different risk factors for uterine rupture like multiparity, congenital uterine anomalies, previous caesarean deliveries, macrosomia, labour induction, uterine instrumentation, uterine trauma and uterine surgery like previous uterine myomectomy. Signs and symptoms of uterine rupture are nonspecific; we can observe fetal distress, a decrease in uterine contractions, abdominal pain, uterine bleeding, maternal hemodynamic changes, etc. A high index of suspicion, rapid diagnosis and early treatment minimizes maternal and fetal mortality. However we must evaluate patients according to their risk and agree on the way of safer childbirth.
\end{abstract}

Keywords: Uterine rupture; Pregnancy; Patient; Labor induction; Prostaglandins; Surgery; Uterus; Vaginal birth

\section{Introduction:}

Uterine rupture during pregnancy is the disruption of the uterine wall. It is a rare complication but it is associated with high maternal and perinatal morbidity and mortality. This has a variable incidence between $0.02-0.08 \%$ of pregnancies [1]. In developing countries, data collection may be difficult, but some series have been published reporting a higher incidence (2-3 per 1000 births) [1,2]. There are different risk factors for uterine rupture like multiparity, congenital uterine anomalies, previous caesarean deliveries and uterine surgery like previous uterine myomectomy, macrosomia, labor induction, uterine instrumentation and uterine trauma.

In developing countries the most common cause is traumatic birth and hyperdynamia, therefore in developed countries is previous caesarean section (Figures 1 and 2) There are American and European guidelines, consensus documents, meta-analyzes, which try to give some guidelines to standardize our medical practice. Some of them review contraindications, risks and make recommendations for achieving vaginal birth after cesarean (VBAC) safely. Previous caesarean delivery has an odds ratio (OR) more than 2 for the rupture of the scarred uterus. Risk of uterine rupture for women who labor spontaneously after a previous caesarean delivery is $0.44 \%$, vs $0.16 \%$ in women who repeat caesarean delivery without trial of labor. In cases oxytocin stimulation is performed, it is about $1-4 \%$, but data are conflicting, because of differences in use of dose and time of stimulation.

Currently uncommon practice of the vertical incision (Classic vertical midline uterine incision). The data collected in studies with this type of incision show a higher rate of uterine rupture associated trial of labor after caesarean (TOLAC) to cases with low transverse incision ( $1.2 \%$ vs $0.53 \%$ in spontaneous TOLAC) The data regarding the risk associated with cervical ripening with prostaglandins are also variable, with rates between $1.3 \%$ to $5.2 \%$ [3] If we analyze data of risk of rupture in patients with multiple caesarean, we find an increased risk of rupture 3 times higher with 2 or more caesarean compared with patients with 1 uterine scar. The uterine rupture associated with previous caesarean may be related to abnormally invasive placenta, when the placenta becomes abnormally adherent to the myometrium rather than the uterine decidua. Rates of placenta accreta are increasing due to the increase in the rate of caesarean delivery. Combination of placenta previa and prior caesarean exponentially increases the risk of placenta accreta.

Other risk factors include uterine curettage, myomectomy, hysteroscopic surgery, pelvic irradiation [4]. The global incidence of placenta accreta is $1 / 2.5$ to $1 / 7$ pregnancies [5]. The uterine rupture associated with accretism is rare, only $1 \%$ of them are attributed to placenta accrete [6] the rupture is extremely rare in the unscarred uterus, there are series with rates of less than $0.01 \%$ of deliveries [3] (Figure 3). 


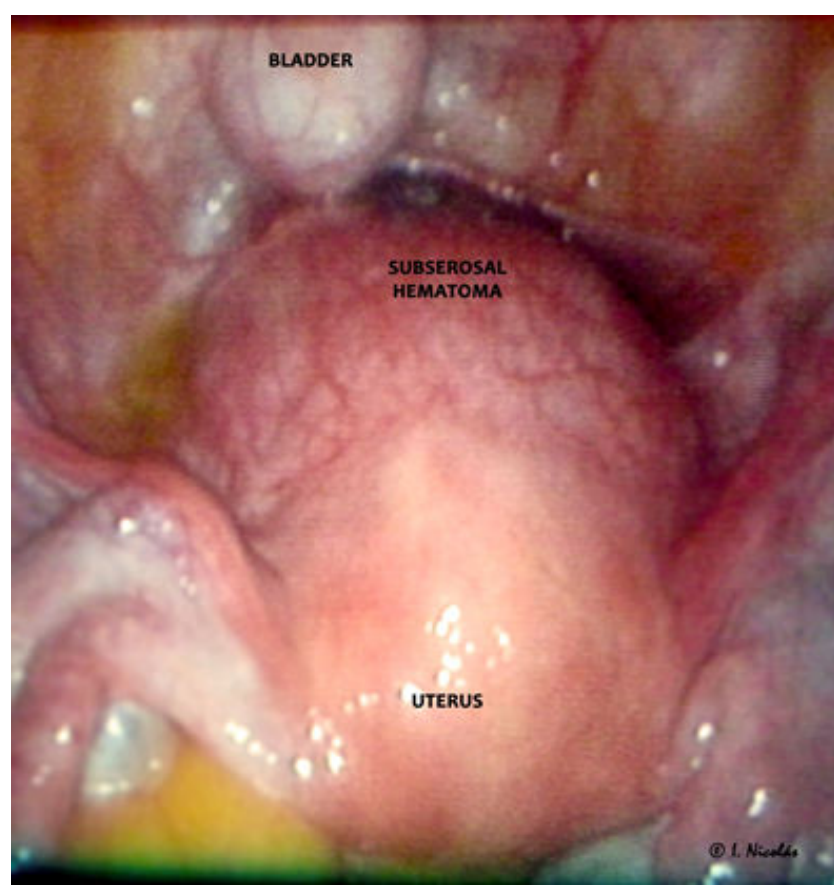

Figure 1: Latrogenic uterine rupture by curettage subsequent spontaneous abortion 14 weeks. We can observe free intraperitoneal blood and subserosal hematoma.

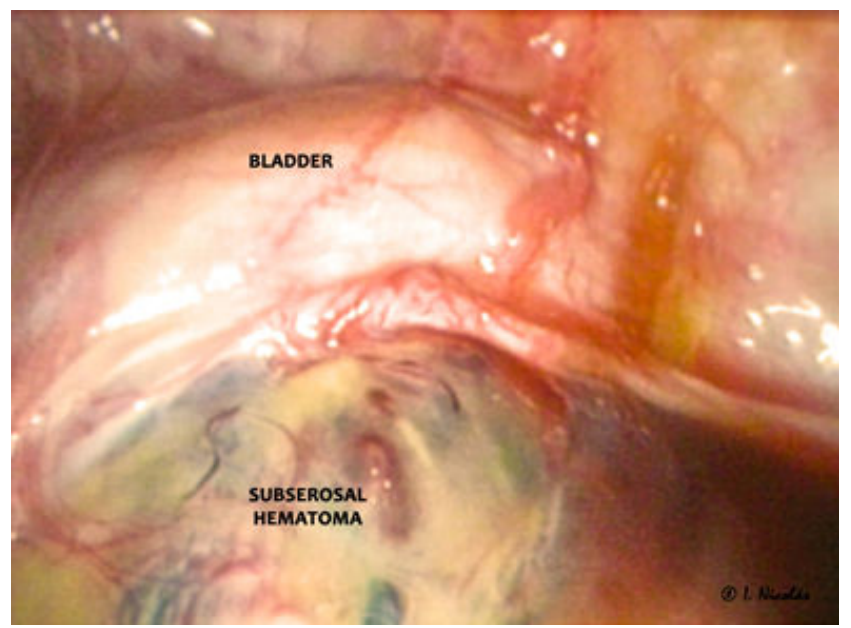

Figure 2: Detail of the injury

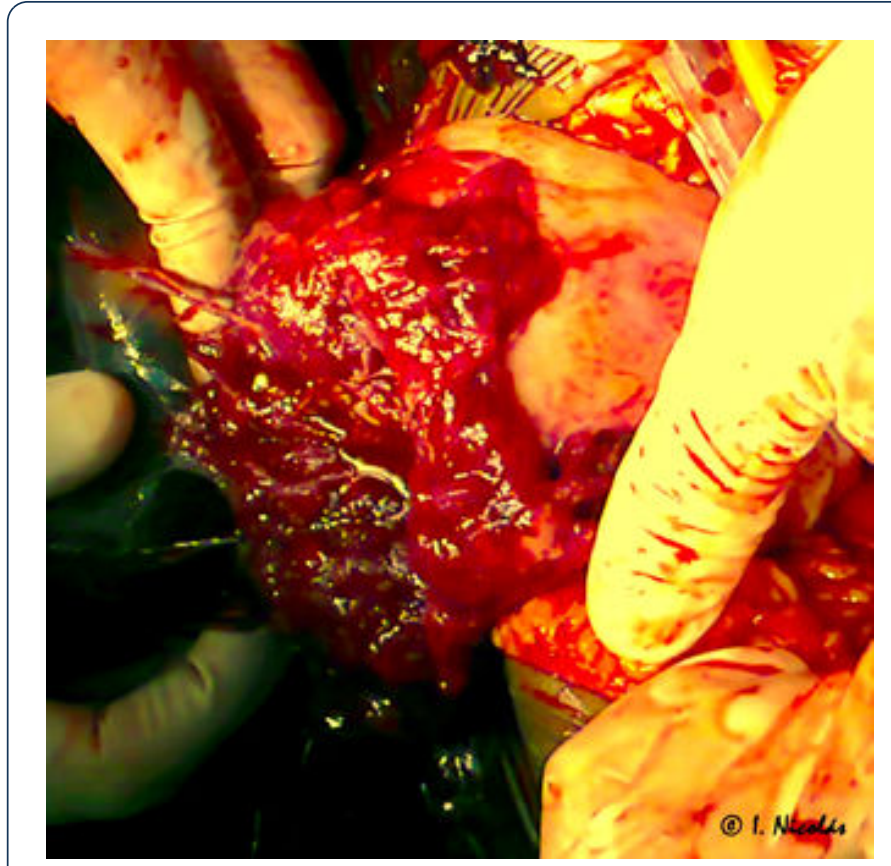

Figure 3: Spontaneous uterine rupture due to placeta accreta

\section{Signs, Symptoms and Diagnosis}

The signs and symptoms of uterine rupture are nonspecific; they are less dramatic in previous uterine scar than a spontaneous rupture. We can observe fetal distress (decelerations, bradycardias can be observed in the no stress test), a decrease in uterine contractions or abnormal patterns of uterine activity like hiperstimulation, abdominal pain, uterine bleeding, recession of the presenting fetal part, maternal hemodynamic changes, shock, etc. [1,3]. A high index of suspicion, rapid diagnosis and prompt treatment minimizes maternal and fetal mortality. It is very difficult to have a quick and conclusive test for diagnosis before surgery, so it is important suspect the uterine rupture on the basis of signs and symptoms.

The ultrasonography can help us in the diagnosis, but we are often in a medical emergency and the time performance is crucial. Some authors have linked the thickness of the wall prior to birth with risk of rupture establishing a cut-off of $3.5 \mathrm{~mm}$ [3]. So in some patients we consider risk, we could raise the antepartum assessment of the uterine wall through ultrasound, to better target the way of actual delivery. Ultrasound can help in the diagnosis of placenta accreta in high risk populations too. In cases of abnormally invasive placenta there may be a loss or disruption in the continuity of the echo lucent myometrial zone, a disruption of the bladder wall or protrusion of the placenta into the bladder, irregular vascular spaces, termed lacunae and increased vascularity in the placenta-bladder interface [7]. The Magnetic Resonance Imaging (MRI) also has been used for the antenatal diagnosis [4]. 


\section{Complications}

The primary risk of uterine rupture is haemorrhage and its consequences. Haemorrhage can trigger a hypovolemic shock, a disseminated intravascular coagulation (DIC) and finally a multiorgan failure in the mother. Blood transfusion is required in a quarter of the cases, a $20 \%$ need approximately hysterectomy, and consequently it implies the impossibility of giving birth again. Some surgical complications can be given, the most common is cystectomy, especially if there are placenta accrete $[3,4,6]$. Less common injuries occur to the ureter and bowel. Some pelvic nerves can be injured causing pelvic pain. Other complications related to surgery and hospitalization is infection, need for a second operation, thromboembolism and vesicovaginal fistula. Maternal death occurs at a rate of $1 \%$ in developed countries and 5-10\% in developing countries [3].

The fetal and neonatal consequences are related with the haemorrhage and the prematurity. Maternal hypovolemia can decrease placental perfusion and compromise fetal oxygenation, so that the neonate can develop hypoxic-ischemic encephalopathy. The degree of neurological damage depends on the time from diagnosis to delivery. The umbilical-artery cord $\mathrm{PH}$ level can show a fetal acidosis, and the APGAR score can be altered in these new-born's. Some of them need intensive cares with prolonged hospital stays and special paediatric care over time. Death rates are different in industrialized countries and developing countries, 7 and $74 \%$ respectively [3].

\section{Treatment}

When the diagnosis is made intrapartum we must stabilize the mother if necessary, an immediate laparotomy and remove the fetus. Fetal mortality and morbidity will be determined by the time it takes to perform the caesarean. The surgical treatment for the mother depends on the extent of the damage, if it is small rupture we can try hysterorraphy, wishes of the woman to future fertility should also be considered, but in cases of maternal destabilization, coagulopathy, extensive damage and difficult control haemorrhage, the hysterectomy is the treatment of choice.

\section{Discussion}

Many guides and obstetric associations reflect the benefit of vaginal birth for the new-born, this being feasible way of delivery in cases of previous caesarean. But it is true that thought the incidence is low, the scientific evidence shows an increase in these cases of uterine rupture. It is our duty to manage labor of these patients, avoiding risks that could enhance the possibility of uterine rupture, such as the hiperdinamias. We have tools that can help us to manage, such as the continuous registration cardiotocographic, internal monitoring that can be useful in cases of obesity or difficulty dynamics control.

There are no reliable diagnostic methods to completely predict risk of uterine rupture. The study and comprehensive approach to the patient can help us make a decision on the future route of delivery. There is no standardized cut-off thickness of the lower segment; some authors make the limit at $3.5 \mathrm{~mm}$, other $2.5 \mathrm{~mm}$. Some perform the measurement with transvaginal and other transabdominal probe. But considering that ultrasound is now part of our regular routine, it is accessible in obstetrical services and it is advisable ultrasound examination in the third trimester, in patients with previous caesarean, evaluation of the lower segment are no extra expense nor additional practice in pregnant and could help in planning delivery $[3,8,9]$. In patients at risk, we must conduct a preliminary study to delivery to evaluate possible factors such as fetal macrosomia, fetal position, placental alterations, number of previous caesarean scars, types of uterine incision.

In our environment the caesarean rate has increased, with risk of uterine rupture associated with the previous scar during the pregnancy. Alterations in the placenta, such as placenta previa and accreta must be observed and studied during pregnancy and if necessary establish a multidisciplinary management and a planned delivery. Adequate obstetric care to these patients, planning delivery, guiding the vaginal birth canal patients with less risk and careful intrapartum control, can help us provide a safe VBAC, offering the neonatal benefit and avoiding unnecessary repeat elective abdominal deliveries. Another point to consider is the time interval between caesarean and vaginal delivery following. We must bear in mind that the short period intergenesic involves an increased risk of rupture, some authors have discouraged vaginal delivery if the interdelivery interval is shorter than 18 months [10].

The use of prostaglandins is controversial in patients with previous caesarean section. American guidelines discourage the use of prostaglandins for cervical ripening and induction in patients with previous caesarean but some European protocols allow use. Some authors have reported the safety of prostaglandin E2, others relate to increased uterine rupture, while for misoprostol use has been reported taxas data with high uterine rupture [11]. Some reviews conclude with the lack of clinical trials, some guidelines contraindicate the use of prostaglandins in patients with previous caesarean (12-15)

It is our obligation to inform patients, agree on delivery and carry out the safest for both the mother and the fetus approach. We know the benefits of vaginal delivery, and we must offer it to patients with previous caesarean showing no apparent risk factors in the prenatal visit. We have to keep in mind the high success rate of vaginal birth after caesarean (74\%, reaching $83 \%$ if there has been a previous vaginal delivery) [16], but we must be aware and cautious in handling delivery of these patients, paying attention to the signs and symptoms of alarm. The safest model would be the patient with one prior low transverse uterine incision, an interval intergenesic more than 18 months, no prior and no alterations observed by ultrasound placenta, no macrosomia and fetus with a cephalic presentation.

The diagnosis of uterine rupture should be fast during labour or patient care in the emergency room, because maternal and fetal morbidity and mortality depend on it. We must be aware of this situation, clearly explain to patients and make the decision together to achieve the best security for the mother and newborn, translating this information in informed consent. 


\section{References}

1. Care protocols of the Spanish Society of Gynecology and Obstetrics (2006) Uterine rupture.

2. Kidantou HL, Mwampagatwa I, Van Roosmalen J (2012) Uterine rupture: a retrospective analysis of causes complications and management outcomes at Muhimbili National Hospital in Dar es Salaam, Tanzania. Tanzan J Health Res 14: 1-8.

3. Nahum G, Pham K (2012) Uterine Rupture in Pregnancy. Medscape.

4. Silver R, Queenan JT, Hobbins JC, Spong CY (2010) Protocols for High-Risk Pregnancies. $5^{\text {th }}$ edn. Inglaterra: Wiley-Blackwell pp: 520-528.

5. Inmaculada Nicolás Perea, Alfredo Hernández Embry, Núria Estopiñá Cros, Walter Nicolás Trusso, Lorena Serrano Martín, et al. (2015) Uterine rupture due to placenta percreta at 17 weeks of pregnancy. Prog Obstet Ginecol 58: 5.

6. Colmorn LB, Petersen KB, Jakobsson M, Lindqvist PG, Klungsoyr K, et al. (2015) The Nordic Obstetric Surveillance Study: a study of complete uterine ruptura, abnormally invasive placenta, peripartum hysterectomy, and severe blood loss at delivery. Acta Obstet Gynecol Scand 94: 734-744.

7. Higueras MT, Cabero LL, Saldıvar D (2013) Hemorragias postparto debidas a problemas adherenciales placentarios. Hemorragias obstetricas. $1^{\text {st }}$ edn, España: Ergon pp: 111-123.

8. Rozenberg P, Goffinet F, Philippe HJ, Nisand I (1999) Thickness of the lower uterine segment: its influence in the management of patients with previous cesarean sections. Eur J Obstet Gynecol Reprod Biol 87: 39-45.
9. Abdel M, Diaa A, Mamdouh H, Enas M (2010) Ultrasonographic evaluation of lower uterine segment thickness in pregnant women with previous cesarean section. Middle East Fertil Soc J 15: 188-193.

10. Bujold E, Gauthier RJ (2010) Risk of uterine rupture associated with an interdelivery interval between 18 and 24 months. Obstet Gynecol 115: 1003-1006.

11. Blanco J, Collins M, Willis D, Prien S (1992) Prostaglandin E2 Gel Induction of Patients with a Prior Low Transverse Cesarean Section. Am J Perinatol 9: 80-83.

12. Jozwiak M, Dodd JM (2013) Methods of term labour induction for women with a previous caesarean section. Cochrane Database of Systematic Reviews 3.

13. American College of Obstetricians and Gynecologists (2010) ACOG Practice bulletin no. 115: Vaginal birth after previous caesarean. Obstet Gynecol 116: 450-463.

14. Blanchette HA, Nayak S, Erasmus S (1999) Comparison of the safety and efficacy of intravaginal misoprostol (prostaglandin E1) with that of dinoprostone (prostaglandin E2) for cervical ripening and induction of labor in a community hospital. Am J Obstet Gynecol 180: 1551-1559.

15. Society of Obstetricians and Gynaecologists of Canada (SOGC) Guidelines for vaginal birth after previous cesarean birth: N 155 (2005) J Obstet Gynaecol Can pp 164-174.

16. Cunningham FG, Bangdiwala S, Brown SS, Dean TM, Frederiksen M (2010) Vaginal birth after cesarean: news insights. National Institute of Health Consensus Development Conferences Statement. Obstet Gynecol 115: 1279-1295. 